\title{
Estudio Madrid sobre prevalencia y características de los pacientes con patología dual en tratamiento en las redes de salud mental y de atención al drogodependiente
}

\section{Madrid study on the prevalence and characteristics of outpatients with dual pathology in community mental health and substance misuse services}

\author{
Francisco Arias ${ }^{*}$; Nestor Szerman ${ }^{\star *}$; Pablo \\ VEGA ${ }^{* \star *}$; BeATRIZ MESIAs ${ }^{\star * *}$; IGNACIO BASURTE*; \\ ConsUelo MoranT ${ }^{\star \star \star *}$; EnRIQUeta OCHOA ${ }^{\star \star \star \star *}$; FéLIX \\ POYO ${ }^{\star \star \star * \star * ; ~ F R A N C I S C O ~ B A B i ́ N ~}{ }^{\star \star \star \star \star \star * ~}$
}

*Servicio de Psiquiatría. Hospital Doce de Octubre. Madrid.

**Servicio de Psiquiatría. Hospital General Gregorio Marañon. Madrid.

${ }^{* \star *}$ Instituto de Adicciones. Madrid.

${ }^{\star * \star *}$ Oficina Regional de Salud Mental. Madrid.

*****Servicio de Psiquiatría. Hospital Ramón y Cajal.

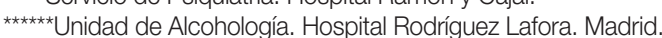

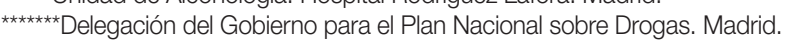

Enviar correspondencia a:

Francisco Arias

CSM Villaverde.

C/ Totanes 1. 28041. Madrid.

TIfno: 917955546

E-mail: farias1012@gmail.com

\section{Resumen}

Se valora la prevalencia y caracteristicas de los pacientes de patología dual (diagnóstico actual de un trastorno mental y de un trastorno por uso de sustancias (TUS)): en las redes asistenciales de Salud Mental y Drogodependencias de la Comunidad de Madrid. Se consigue una muestra de 837 sujetos (208 de la red de Salud Mental y 629 de la red de Drogodependencias). Se usó la entrevista MINI (Mini International Neuropsychiatric Interview) y el cuestionario PD04+ (Personality Disorder Questionnaire) para la valoración de los trastornos del eje I y II. Se hallaron $517(61,8 \%)$ pacientes con patología dual (un $36,1 \%$ en la red de salud mental y un 70,3\% en la red de drogas). Al compararlos con el grupo de sujetos con TUS sin patología dual $(n=194)$, habia entre los duales menos varones y peor situación laboral, siendo las drogas más consumidas el alcohol y cannabis. Al compararlos con el grupo de trastornos mentales sin uso de sustancias $(n=126)$, encontramos diferencias en todas las caracteristicas sociodemográficas analizadas y los casos de patología dual son diagnosticados más frecuentemente como trastorno bipolar, agorafobia, trastorno por ansiedad generalizada, trastorno por estrés postraumático, mayor riesgo de suicidio y distintos trastornos de personalidad. Por lo tanto, la presencia de patología dual es elevada en sujetos en tratamiento y presentan unas características diferenciales, tales como mayor riesgo de suicidio y situación laboral precaria que hacen pensar en un peor pronóstico, cuestión a considerar para el desarrollo de recursos asistenciales adecuados.

Palabras clave: Dependencia de drogas, patología dual, trastornos de personalidad, trastorno bipolar, trastornos de ansiedad. recibido: Jullio 2012 aceptado: Diciembre 2012

\section{Abstract}

The objective was to quantify the prevalence of dual diagnosis and to evaluate the characteristics of these patients from community mental health and substance misuse services in Madrid. The sample consisted of 837 outpatients from Madrid, 208 from mental health services and 629 from substance misuse services. We used the Mini International Neuropsychiatric Interview (MINI) and Personality Disorder Questionnaire (PD04+) to evaluate disorders from axis I and II. It was considered that 517 (61.8\%) patients had dual pathology (current diagnoses of axis I or II disorders and an addictive disorder): $36,1 \%$ in mental health services and $70,3 \%$ in substance misuse services. There were fewer males amongst the dual patients and it was also found that they had a worse employment situation, along with higher figures of alcohol and cannabis dependence than addicts without dual diagnoses $(n=194)$. When comparing them with patients with mental disorder diagnoses only, excluding substance use disorder $(n=126)$, there were differences in all socio-demographic characteristics analyzed, and dual patients were associated with diagnoses of bipolar disorder, agoraphobia, generalized anxiety disorder, post-traumatic stress disorder, and had more suicide risk and different personality disorders. Thus, dual pathology is higher in patients who are in treatment and have differential characteristics (higher suicide risk, worse employment situation) that suggest greater severity that could be of help in the planning of care resource policies for these patients.

Key Words: Drug dependence, dual diagnosis, personality disorders, bipolar disorder, anxiety disorders. 
L a patología dual se define como la presencia de un trastorno adictivo y un trastorno mental en un individuo, dentro de un periodo concreto (Volkow, 2007). Dicha comorbilidad es habitual como señalan importantes estudios epidemiológicos en población general (Compton, Thomas, Stinson y Grant, 2007; Kessler, Chiu, Demler, Merikangas y Walters, 2005; Regier et al., 1990), e igualmente en muestras clínicas (Chan, Dennis y Funk, 2008; Weaver et al., 2003). La comorbilidad se asocia con un mayor uso de servicios sanitarios, fenotipos distintos, mayor gravedad del cuadro, mayor discapacidad funcional y peor curso evolutivo (Burns y Teesom, 2002; 0'Brien, Charney et al. 2004; Weaver et al., 2003). Los mecanismos que justifican esta comorbilidad son complejos. Intervienen factores neurobiológicos, genéticos y ambientales que se superponen en la patogenia de estos trastornos (Volkow, 2004; 2007).

Disponemos de escasos datos de la prevalencia de patología dual (PD) en población española y sobre la distribución de esta entre las dos redes asistenciales principales en su asistencia, la red de drogas y la de salud mental (González-Sáiz et al., 2011; Gual, 2007; Pedrero-Pérez, Puerta-García, Lagares y SáezMaldonado A., 2003; Torrens, Gilchrist y Domingo-Salvany, 2011; Vergara-Moragues et al., 2012). El conocimiento sobre la distribución de la PD y las características de estos sujetos puede ayudar a optimizar los recursos sanitarios. En el estudio piloto sobre el estudio de la prevalencia de PD realizado por la Sociedad Española de Patología Dual (SEPD) se detectó un $34 \%$ de prevalencia de esta, siendo mayor en la red de atención al drogodependiente que en la de salud mental (Szerman et al, 2011). Dicho estudio piloto fue un estudio retrospectivo basado en los datos de la historia clínica de los pacientes, con las limitaciones propias de este tipo de estudios, pero sirvió para diseñar un estudio metodológicamente más potente, que es él que ahora presentamos, seleccionando las variables más informativas, incorporando una entrevista semiestructurada y con una muestra más amplia. El objetivo principal fue cuantificar la prevalencia de los distintos tipos de patologías incluidas dentro del complejo "patología dual" (trastorno mental y trastorno por consumo de sustancias) en las dos redes asistenciales implicadas en su atención de la Comunidad de Madrid y analizar las características de este grupo de pacientes. El diseño y resultados referidos al riesgo de suicidio de los pacientes duales han sido previamente publicados (Szerman et al, 2011). En el presente trabajo presentamos los datos referentes a la prevalencia de PD en las dos redes asistenciales y a las características sociodemográficas y clínicas diferenciales entre los pacientes duales y aquellos con diagnósticos "puros" de trastorno por uso de sustancias (TUS) y aquellos con diagnóstico exclusivo de trastornos mentales (TM).

\section{Método}

\section{Participantes}

Los pacientes fueron seleccionados consecutivamente por sus propios terapeutas en los centros de tratamiento de drogas
(CAID y CAD: Centros de atención a las drogodependencias de la Comunidad de Madrid y del Ayuntamiento de Madrid respectivamente, en adelante CD-Centros de Drogodependencias) y en los centros de salud mental (CSM) de la Comunidad de Madrid que participaron. A todos estos centros de la Comunidad de Madrid se les propuso la posibilidad de participar con un investigador por centro y entre 10-20 pacientes por centro. Por lo tanto, no había una selección aleatoria de los centros ni de los participantes. Los criterios de inclusión eran pacientes que acudieran a esos centros para una primera valoración 0 estuvieran en seguimiento en dichos centros y fueran mayores de 18 años. Participaron 81 entrevistadores (psiquiatras, psicólogos o médicos generales con amplia experiencia en adicciones) de 64 centros de drogas de la Comunidad de Madrid y de 17 centros de salud mental. Todos los entrevistadores (personal del propio centro) recibieron entrenamiento en la administración de los instrumentos utilizados en el estudio. Los participantes firmaron un consentimiento informado. El estudio fue aprobado por el Comité de Investigación Ética del Hospital Gregorio Marañon de Madrid. El porcentaje de participación fue del $87,2 \%$.

Se incluyeron 837 pacientes: 208 (24,9\%) procedian de CSM y $629(75,1 \%)$ de CD. Del total de 837 pacientes evaluados, $517(61,8 \%)$ se consideraron que tenían PD (diagnóstico actual de un trastorno mental distinto al uso de sustancias y/o un trastorno de personalidad y un diagnóstico de TUS), de estos 442 procedían de los CD (70,3\% de prevalencia de PD en CD) y 75 procedian de CSM (36,1\% de prevalencia de PD en la red de salud mental). El grupo de pacientes duales se comparó con el grupo de sujetos con diagnóstico de TUS no duales ( $n=194,23,2 \%$ de la muestra) y con el grupo de pacientes con diagnóstico de TM no dual ( $n=126,15,1 \%$ de la muestra).

\section{Instrumentos}

Se elaboró un Cuaderno de Recogida de Datos considerando las aportaciones recogidas en el estudio piloto mencionado sobre las variables más informativas y de mayor interés clínico en el momento del análisis. Para establecer la presencia de trastornos mentales se utilizó la entrevista estructurada Mini International Neuropsychiatric Interview (MINI) que permite diagnósticos según criterios DSM-IV y CIE-10 (Sheehan et al., 1998; versión 5,0, Fernando, Bobes, Gibert, Soto y Soto, 2000). Es una entrevista que permite explorar los principales trastornos psiquiátricos del eje I actuales y a lo largo de la vida. Para aquellos trastornos mentales que dicho instrumento no valora a lo largo de la vida, estos se exploraron a través de la entrevista clínica.

Para establecer el diagnóstico de los trastornos de personalidad (TP) se utilizó la escala PDQ4+ (Personality Disorder Questionnaire) (Adaptación Española de Calvo, Caseras, Gutierrez y Torrubia, 2002). Este instrumento combina la rapidez y comodidad de uso de un cuestionario autoadministrado con el control del efecto de la sintomatología de estado de una entrevista. Dicho instrumento consta de una parte autoinformada y una parte heteroadministrada (la escala de significación clínica) que permite confirmar o no los resultados del autoinforme. Se usaron ambas partes. Es un buen instrumento de cribaje a 
nivel clínico, se comporta como una buena herramienta diagnóstica siguiendo los criterios DSM-IV cuando se administra la escala de significación clínica.

\section{Análisis estadístico}

Se calcularon en todas la variables los parámetros descriptivos: media y desviación estándar en las cuantitativas que se ajusten a una distribución normal (test de Shapiro-Wilk) y en las que no se ajustan, la mediana y el rango intercuartílico. Las variables cualitativas se expresan mediante frecuencias relativas porcentuales. La comparación entre dos grupos se realizó mediante la prueba de chi-cuadrado $\left(\chi^{2}\right)$ o la prueba exacta de Fischer si procedía para las variables categóricas y mediante la prueba t de Student o la U de Mann-Whitney para las variables cuantitativas. La variable principal se calcula en el intervalo de confianza para un $95 \%$ de seguridad. Los test se consideran significativos si la $p<0,05$. En la comparación entre diagnósticos del eje I y II entre pacientes duales y no duales, se calcularon las Odds ratio no ajustadas y sus intervalos de confianza al 95\% para aquellas comparaciones que resultaron estadísticamente significativas. El análisis estadístico se genera mediante el programa SPSS v.17.

\section{Resultados}

La tabla 1 recoge las características sociodemográficas de los pacientes de los tres grupos analizados. Los sujetos duales presentaban menor porcentaje de varones y peor situación laboral que aquellos con TUS no duales. Había diferencias significativas en todas las variables analizadas entre el grupo de duales y aquellos con TM no duales. Los pacientes duales respecto a los pacientes con TM eran más jóvenes, con mayor predominio de varones, vivían con su familia de origen, tenían peor nivel educativo y peor situación laboral y mayor prevalencia de hepatitis $\mathrm{B}$ ó $\mathrm{C}$ e infección por el virus de la inmunodeficiencia adquirida (VIH).

\section{Comparación entre los pacientes duales y adictos no duales}

Como se refleja en la Tabla 2, los pacientes duales no tenían una mayor prevalencia de trastorno por uso de alcohol actual respecto a los adictos no duales, aunque si se consideraba el diagnóstico de trastorno por uso de alcohol a lo largo de la vida, la diferencia se hacía estadísticamente significativa (396 de los duales, 76,6\%, frente a 132 de los adictos no duales, $68,0 \%, p=0,02$ ). No había diferencias en la frecuencia de otros TUS. Los pacientes duales presentaban mayor gravedad del TUS de alcohol y de cannabis, con mayor presencia de pacientes con diagnóstico de dependencia de estas sustancias que de abuso.

No se observó una diferencia en el número de consumos entre los duales y los adictos puros (prueba $U$ de Mann-Whitney, $p=0,07)$. No había diferencias en la edad referida de inicio de las distintas sustancias entre ambos grupos (Tabla 2).
Tabla 1. Características sociodemográficas de los pacientes con y sin diagnóstico dual

\begin{tabular}{|c|c|c|c|c|c|}
\hline & $\begin{array}{l}\text { Pacientes } \\
\text { duales } \\
(n=517)\end{array}$ & $\begin{array}{l}\text { Pacientes } \\
\text { con trastor- } \\
\text { no por uso } \\
\text { de sustancias } \\
(n=194)\end{array}$ & $\begin{array}{l}\text { P (duales } \\
\text { vs TUS) }\end{array}$ & $\begin{array}{c}\text { Pacientes } \\
\text { con trastor- } \\
\text { no mental } \\
(n=126)\end{array}$ & $\begin{array}{c}\text { P (duales } \\
\text { vs TM } \\
\text { no } \\
\text { duales) }\end{array}$ \\
\hline Edad (años) & $37,1(\mathrm{DE}=9,3)$ & $37,7(8,6)$ & NS & $44,3(13,3)$ & 0,001 \\
\hline Varón & 403 (78,1\%) & $174(90,2 \%)$ & 0,001 & $40(31,7 \%)$ & 0,001 \\
\hline \multicolumn{6}{|l|}{ Estado civil } \\
\hline Soltero & 301 (59,7\%) & $104(53,9 \%)$ & NS & $49(38,9 \%)$ & 0,001 \\
\hline Casado & $117(23,2 \%)$ & $59(30,6 \%)$ & & $57(45,2 \%)$ & \\
\hline $\begin{array}{l}\text { Divorciado/ } \\
\text { viudo }\end{array}$ & $86(17,1 \%)$ & $30(15,5 \%)$ & & $20(15,9 \%)$ & \\
\hline \multicolumn{6}{|l|}{ Convivencia } \\
\hline Solo & $82(15,9 \%)$ & $20(10,4 \%)$ & NS & $19(15,1 \%)$ & 0,001 \\
\hline Familia propia & $142(27,6 \%)$ & $68(35,2 \%)$ & & $65(51,6 \%)$ & \\
\hline $\begin{array}{l}\text { Familia de } \\
\text { origen }\end{array}$ & $227(44,1 \%)$ & $80(41,5 \%)$ & & $38(30,2 \%)$ & \\
\hline $\begin{array}{l}\text { Institucionali- } \\
\text { zado }\end{array}$ & $20(3,9 \%)$ & $10(5,2 \%)$ & & 0 & \\
\hline $\begin{array}{l}\text { Otros (con } \\
\text { amigos, en la } \\
\text { calle...) }\end{array}$ & $44(8,5 \%)$ & $15(7,8 \%)$ & & $4(3,2 \%)$ & \\
\hline \multicolumn{6}{|l|}{ Educación } \\
\hline Sin estudios & $6(1,2 \%)$ & $1(0,5 \%)$ & NS & $6(4,8 \%)$ & 0,001 \\
\hline Primarios & $239(46,8 \%)$ & $96(49,5 \%)$ & & $35(28,2 \%)$ & \\
\hline Secundarios & $198(38,7 \%)$ & $73(37,6 \%)$ & & $41(33,1 \%)$ & \\
\hline Universitarios & $60(11,7 \%)$ & $23(11,9 \%)$ & & $42(33,9 \%)$ & \\
\hline \multicolumn{6}{|l|}{$\begin{array}{l}\text { Situación } \\
\text { laboral }\end{array}$} \\
\hline Activo & 228 (44,3\%) & $105(54,1 \%)$ & 0,02 & $70(55,6 \%)$ & 0,001 \\
\hline Sin empleo & $173(33,6 \%)$ & $67(34,5 \%)$ & & $17(13,5 \%)$ & \\
\hline Incapacitado & $68(13,2 \%)$ & $10(5,2 \%)$ & & $14(11,1 \%)$ & \\
\hline $\begin{array}{l}\text { Otros (estu- } \\
\text { diantes, sus } \\
\text { labores...) }\end{array}$ & $46(8,9 \%)$ & $12(6,1 \%)$ & & $25(19,8 \%)$ & \\
\hline $\begin{array}{l}\text { Patología } \\
\text { médica }\end{array}$ & 184 (35,8\%) & $76(39,2 \%)$ & NS & $42(33,3 \%)$ & NS \\
\hline VIH & $52(10,1 \%)$ & $24(12,4 \%)$ & NS & 0 & 0,001 \\
\hline Hepatitis B o C & $113(21,9 \%)$ & $58(29,9 \%)$ & 0,03 & $3(2,4 \%)$ & 0,001 \\
\hline
\end{tabular}

TUS=Trastorno por uso de sustancias. TM= Trastornos mentales. De=desviación estándar. VIH= Virus de la Inmunodeficiencia Humana. 
Tabla 2. Diferencias en uso de drogas entre pacientes duales y adictos no duales.

\begin{tabular}{|c|c|c|c|}
\hline Diagnóstico & $\begin{array}{l}\text { Duales } \\
(\mathrm{n}=517) \\
\mathrm{n}(\%)\end{array}$ & $\begin{array}{l}\text { Trastorno por uso } \\
\text { de sustancias } \\
\text { (n=194) } \\
n(\%)\end{array}$ & $p$ \\
\hline \multicolumn{4}{|l|}{$\begin{array}{l}\text { Diagnóstico actual de } \\
\text { Trastorno por uso de } \\
\text { sustancias }\end{array}$} \\
\hline $\begin{array}{l}\text { Trastorno por uso de alcohol } \\
\text { Dependencia } \\
\text { Abuso }\end{array}$ & $\begin{array}{c}263(50,9 \%) \\
196(74,5 \%) \\
67(25,5 \%)\end{array}$ & $\begin{array}{l}84(43,3 \%) \\
50(59,5 \%) \\
34(40,5 \%)\end{array}$ & $\begin{array}{c}0,07 \\
0,008\end{array}$ \\
\hline $\begin{array}{l}\text { Trastorno por uso de cocaina } \\
\text { Dependencia } \\
\text { Abuso }\end{array}$ & $\begin{array}{c}274(53,0 \%) \\
242(88,3 \%) \\
32(11,7 \%)\end{array}$ & $\begin{array}{l}91(46,9 \%) \\
77(84,6 \%) \\
14(15,4 \%)\end{array}$ & $\begin{array}{l}\text { NS } \\
\text { NS }\end{array}$ \\
\hline $\begin{array}{l}\text { Trastorno por uso de } \\
\text { opiáceos } \\
\text { Dependencia } \\
\text { Abuso }\end{array}$ & $\begin{array}{c}67(13,0 \%) \\
63(94 \%) \\
4(6 \%)\end{array}$ & $\begin{array}{c}33(17,0 \%) \\
32(97 \%) \\
1(3 \%)\end{array}$ & NS \\
\hline $\begin{array}{l}\text { Trastorno por uso de can- } \\
\text { nabis } \\
\text { Dependencia } \\
\text { Abuso }\end{array}$ & $\begin{array}{l}103(19,9 \%) \\
75(72,8 \%) \\
28(27,2 \%)\end{array}$ & $\begin{array}{l}30(15,5 \%) \\
15(50,0 \%) \\
15(50,0 \%) \\
\end{array}$ & $\begin{array}{l}\text { NS } \\
0,02\end{array}$ \\
\hline Sedantes & $30(5,8 \%)$ & $7(3,6 \%)$ & NS \\
\hline \multicolumn{4}{|l|}{$\begin{array}{l}\text { Edad inicio en consumo } \\
\text { Años (D.E.) }\end{array}$} \\
\hline Alcohol & $16,1(5,9)$ & $16,5(5,2)$ & NS \\
\hline Cocaina & $20,7(6,5)$ & $21,7(6,3)$ & NS \\
\hline Cannabis & $16,1(4,2)$ & $16,4(4,6)$ & NS \\
\hline Opiáceos & $20,0(6,4)$ & $19,4(4,1)$ & NS \\
\hline $\begin{array}{l}\text { Suma de consumos* } \\
\text { Número (D.E.) }\end{array}$ & $1,5(1,0)$ & $1,3(1,0)$ & 0,07 \\
\hline
\end{tabular}

D.E.=desviación estándar. *Prueba U de Mann-Whitney.

\section{Comparación entre los pacientes duales y los sujetos con diagnóstico exclusivo de trastorno mental distinto a las adicciones}

Los pacientes duales tenían mayor número de diagnósticos en el eje I y en el eje II (Tabla 3). Los TM más asociados al diagnóstico dual fueron el trastorno bipolar y distintos trastornos de ansiedad. Los sujetos duales tenían mayor prevalencia de TP y eran estadisticamente más frecuentes los diagnósticos de diversos TP: paranoide, esquizoide, antisocial y límite que en los pacientes con TM no duales. Además los pacientes duales tenían mayor riesgo de suicidio valorado por la MINI y este tendía a ser de mayor gravedad que los sujetos con TM no dual.
Tabla 3. Diferencias en diagnósticos de trastornos mentales actuales y trastornos de personalidad entre pacientes duales y pacientes con trastornos mentales del eje I no duales.

\begin{tabular}{|c|c|c|c|c|c|}
\hline Diagnóstico & $\begin{array}{c}\text { Duales } \\
(n=517) \\
n(\%)\end{array}$ & $\begin{array}{c}\text { Trastorno } \\
\text { mental } \\
(n=126) \\
n(\%)\end{array}$ & $\mathrm{p}$ & $\mathrm{OR}$ & $\begin{array}{c}\text { IC } \\
(95 \%)\end{array}$ \\
\hline \multicolumn{6}{|l|}{ Eje I: Diagnóstico actual* } \\
\hline Episodio depresivo mayor & $160(30,9 \%)$ & $49(38,9 \%)$ & 0,1 & & \\
\hline Distimia & $114(22,1 \%)$ & $24(19,0 \%)$ & NS & & \\
\hline Trastorno bipolar & $162(31,3 \%)$ & $8(6,3 \%)$ & 0,001 & 4,9 & $2,5-9,8$ \\
\hline Trastorno por angustia & $151(29,2 \%)$ & $28(22,2 \%)$ & NS & & \\
\hline Agorafobia & $73(14,1 \%)$ & $8(6,3 \%)$ & 0,02 & 2,2 & $1,1-4,5$ \\
\hline Fobia social & $67(13,0 \%)$ & $10(7,9 \%)$ & NS & & \\
\hline $\begin{array}{l}\text { Trastorno obsesivo- } \\
\text { compulsivo }\end{array}$ & $51(9,9 \%)$ & $17(13,5 \%)$ & NS & & \\
\hline $\begin{array}{l}\text { Trastorno por estrés pos- } \\
\text { traumático }\end{array}$ & $31(6,0 \%)$ & $1(0,8 \%)$ & 0,01 & 7,5 & $\begin{array}{l}1,1- \\
54,8\end{array}$ \\
\hline Psicosis & $59(11,4 \%)$ & $18(14,3 \%)$ & NS & & \\
\hline Bulimia & $8(1,5 \%)$ & $5(4,0 \%)$ & NS & & \\
\hline $\begin{array}{l}\text { Trastorno por ansiedad } \\
\text { generalizada }\end{array}$ & $138(26,7 \%)$ & $18(14,3 \%)$ & 0,004 & 1,9 & $1,2-2,9$ \\
\hline \multicolumn{6}{|l|}{$\begin{array}{l}\text { Riesgo suicidio (valorado } \\
\text { por MINI) }\end{array}$} \\
\hline Presencia riesgo suicidio & $208(40,2 \%)$ & $21(16,7 \%)$ & 0,001 & 2,4 & $1,6-3,6$ \\
\hline Leve & $127(62,0 \%)$ & $15(71,4 \%)$ & & & \\
\hline Moderado & $26(12,7 \%)$ & $5(23,8 \%)$ & 0,07 & & \\
\hline Grave & $52(25,4 \%)$ & $1(4,8 \%)$ & & & \\
\hline \multicolumn{6}{|l|}{$\begin{array}{l}\text { Diagnósticos eje I mul- } \\
\text { tiples** }\end{array}$} \\
\hline $\begin{array}{l}\text { Cualquier trastorno del } \\
\text { humor }\end{array}$ & $329(63,6 \%)$ & $72(57,1 \%)$ & NS & & \\
\hline $\begin{array}{l}\text { Cualquier trastorno de } \\
\text { ansiedad }\end{array}$ & $297(57,4 \%)$ & $53(42,1 \%)$ & 0,002 & 1,4 & $1,1-1,7$ \\
\hline $\begin{array}{l}\text { Suma de trastornos } \\
\text { del eje I } \\
\text { Media (d.e) }\end{array}$ & $2,0(1,6)$ & $1,5(1,2)$ & 0,001 & & \\
\hline \multicolumn{6}{|l|}{ Eje $\|^{* * *}$} \\
\hline $\begin{array}{l}\text { Cualquier Trastorno de } \\
\text { personalidad }\end{array}$ & $346(66,9 \%)$ & $59(46,8 \%)$ & 0,001 & 1,4 & $1,2-1,8$ \\
\hline T. paranoide & $124(24,0 \%)$ & $13(10,3 \%)$ & 0,001 & 2,3 & $1,4-4,0$ \\
\hline T. esquizoide & $48(9,3 \%)$ & $4(3,2 \%)$ & 0,02 & 2,9 & $1,1-7,9$ \\
\hline T. esquizotípico & $67(13,0 \%)$ & $10(7,9 \%)$ & NS & & \\
\hline T. antisocial & $100(19,3 \%)$ & $1(0,8 \%)$ & 0,001 & 24,4 & $3,4-173$ \\
\hline T. limite & $132(25,5 \%)$ & $18(14,3 \%)$ & 0,007 & 1,8 & $1,1-2,8$ \\
\hline T. histriónico & $40(7,7 \%)$ & $7(5,6 \%)$ & NS & & \\
\hline T. narcisista & $46(8,9 \%)$ & $9(7,1 \%)$ & NS & & \\
\hline T. por evitación & $130(25,1 \%)$ & $23(18,3 \%)$ & NS & & \\
\hline T. dependiente & $59(11,4 \%)$ & $12(9,5 \%)$ & NS & & \\
\hline T. obsesivo & $124(24,0 \%)$ & $27(21,4 \%)$ & NS & & \\
\hline T. pasivo-agresivo & $69(13,3 \%)$ & $10(7,9 \%)$ & 0,1 & & \\
\hline T. depresivo & $144(27,9 \%)$ & $33(26,2 \%)$ & NS & & \\
\hline $\begin{array}{l}\text { Cualquier T. personalidad } \\
\text { del Cluster A }\end{array}$ & $173(33,5 \%)$ & $17(13,5 \%)$ & 0,001 & 2,5 & $1,6-3,9$ \\
\hline $\begin{array}{l}\text { Cualquier T. personalidad } \\
\text { del Cluster B }\end{array}$ & $206(39,8 \%)$ & $24(19,0 \%)$ & 0,001 & 2,1 & $1,4-3,0$ \\
\hline $\begin{array}{l}\text { Cualquier T. personalidad } \\
\text { del Cluster C }\end{array}$ & $217(42,0 \%)$ & $42(33,0 \%)$ & 0,08 & 1,3 & $\begin{array}{c}0,97- \\
1,6\end{array}$ \\
\hline $\begin{array}{l}\text { Suma de trastornos del } \\
\text { eje II } \\
\text { Media (d.e) }\end{array}$ & $2,1(2,4)$ & $1,3(2,2)$ & 0,001 & & \\
\hline
\end{tabular}

Nota $-\mathrm{T}=$ Trastorno. NS=No significativo. De=desviación estándar. ${ }^{*}$ Excluye Trastorno por uso de sustancias. ${ }^{*}$ T. del humor incluye: Episodio depresivo mayor, Distimia, Trastorno bipolar. T. de ansiedad incluye: Trastorno por angustia, Agorafobia, Fobia social, Trastorno obsesivo-compulsivo, Trastorno por estrés postraumático y Trastorno por ansiedad generalizada. ${ }^{* *}$ T. personalidad del Cluster A incluye paranoide, esquizoide y esquizotípico. Cluster B incluye antisocial, limite, histriónico, narcisista. Cluster C: evitación, dependiente, obsesivo. 


\section{Discusión}

Para nuestro conocimiento los datos del presente estudio ofrecen información sobre la prevalencia y las características de los pacientes con PD en una de las muestras en tratamiento más numerosa de nuestro país y con implicación de un alto número de centros participantes distribuidos por toda la Comunidad de Madrid. Los datos no son extrapolables a los sujetos en tratamiento en otros recursos asistenciales (atención primaria, pacientes ingresados, recursos de rehabilitación) donde la prevalencia puede diferir notablemente y tampoco a población general, dado que existe acuerdo en que la comorbilidad entre trastornos mentales y adictivos favorece la búsqueda de tratamiento (Compton et al., 2007). Igualmente la presencia de policonsumos es lo habitual en estas muestras y eso dificulta establecer relaciones especificas entre trastornos mentales y adictivos (Kessler, 2004).

\section{Prevalencia de patología dual en sujetos en tratamiento en salud mental y en centros de drogodependencias}

Si en el estudio piloto realizado por nosotros (Szerman et al., 2011) en base a la información obtenida de la historia clínica obtuvimos una cifra de prevalencia de PD del 34\% para el global de la muestra, cuando se mejora la metodología a través del uso de una entrevista estructurada, observamos que dicha cifra claramente infravalora la magnitud del problema. Por lo tanto, un porcentaje importante de pacientes duales no están diagnosticados correctamente, lo que puede sugerir que alguno de los trastornos comórbidos no está detectado y, por lo tanto, no está tratado. Un alto porcentaje de pacientes duales no identificados como tales se ha descrito en otras muestras de sujetos en tratamiento (Weaver et al., 2003).

La magnitud del problema es distinta en las dos redes asistenciales estudiadas. En los CD la mayoría de los pacientes tienen un TM comórbido, lo que sugiere que los profesionales de estos centros deben de estar adecuadamente preparados para la detección y manejo de dichos trastornos mentales. En la red de salud mental la prevalencia de PD es notablemente inferior pero, de cualquier forma, más de una tercera parte de estos pacientes se pueden considerar duales, por lo que es recomendable el cribado sistemático de consumo de drogas en este recurso.

La elevada prevalencia de PD se ha descrito en muestras amplias procedentes de población general (Compton et al., 2007; Kessler et al., 2005; Regier et al., 1990) y en las redes asistenciales de drogas y salud mental (Chan et al., 2008; Ross, Glaser y Germanson, 1988; Rush y Koegl, 2008; Torrens et al., 2011; Weaver et al., 2003). Así se han referido cifras similares a las nuestras, sobre $65-85 \%$ de prevalencia de TM entre adictos en tratamiento (Chan et al., 2008; Ross et al., 1988; Weaver et al., 2003) o un 45\% de TUS entre pacientes psiquiátricos (Weaver et al., 2003).

\section{Diferencias entre pacientes duales y adictos sin comorbilidad psiquiátrica}

Los pacientes duales respecto a sus características sociodemográficas son mas parecidos a los adictos puros que a los pacientes con TM no duales. De cualquier forma existen algunas diferencias relevantes. Hay mayor predominio de mujeres entre los pacientes duales y tienen peor situación laboral, esto último sugerente de una mayor gravedad atribuible a la presencia del diagnóstico dual, diferencias constatadas en la literatura (Burns y Teesom, 2002).

Observamos que la droga mas asociada con la presencia de PD es el alcohol. Otros autores igualmente encontraron que el alcohol y, en algunos estudios el cannabis, se asociaba más con la comorbilidad psiquiátrica que la dependencia de cocaína o heroína (Gual, 2007; Lehman, Myers, Thompson y Corty, 1993; Ross et al., 1988; Weaver et al., 2003). Sin embargo, no todos los trabajos coinciden en los mismos datos y señalaron que la dependencia de cocaína presentaba mayores cifras de comorbilidad (Regier et al., 1990). Este estudio se realizó en población general, por lo que una posible explicación es que el abuso de alcohol en población general se asocie a menor psicopatología que en adictos en tratamiento. En nuestra muestra los adictos con menos prevalencia de patología comórbida fueron los consumidores de opiáceos, posiblemente por llevar bastantes años en tratamiento y encontrarse abstinentes y estos formaban parte importante del subgrupo de adictos sin consumo de alcohol. Otros autores también han señalado una mayor prevalencia de TP en servicios de asistencia a adictos al alcohol que en centros de asistencia a otras drogas (BowdenJones et al., 2004).

Al igual que sugieren otros estudios (Compton et al., 2007; Grant et al., 2004; Kessler, 2004; Marsden, Gossop, Stewart, Rolfe y Farell, 2000; Ross et al., 1988), las formas mas graves del TUS es la mas asociada con la presencia de PD. Así hemos observado que la dependencia de alcohol o de cannabis se asocia con el diagnóstico dual más que la presencia de abuso de estas drogas. Aunque no lo hemos observado para los trastornos por uso de cocaína o de heroína, la escasa presencia de formas más leves de estos trastornos, es decir abuso de cocaína y heroína, podría justificar este dato.

También observamos una tendencia a una asociación entre policonsumo de drogas y presencia de PD, como se ha señalado por otros autores (Agrawal, Lynskey, Madden, Bucholz y Heath, 2007; Kandel, Huang y Davies, 2001; Marsden et al., 2000; Ross et al., 1988). No podemos establecer una relación de causalidad, si el policonsumo favorece la presencia de trastornos mentales o estos últimos son un factor de riesgo para implicarse en el consumo de más sustancias.

En contra de lo esperado, no existían diferencias en la edad de inicio en el consumo entre ambos grupos. Aunque los pacientes duales referian una edad menor en el inicio de alcohol, cocaína y cannabis, estas diferencias no eran significativas. Posiblemente la forma de recogida de esta variable no era adecuada, dado que se realizó a través de la entrevista con el paciente, lo que puede estar sometido a un sesgo de memoria. Existe bastante literatura apoyando que una edad de inicio más precoz en el consumo de ciertas drogas favorece la aparición de ciertos trastornos mentales, por ejemplo entre el consumo de cannabis y la psicosis (Dragt et al., 2012). De cualquier forma esto podría ser válido para los trastornos mentales secundarios al consumo. Es posible que en esta muestra predominaran los trastornos mentales primarios y de ahí que 
no se observaran diferencias. La PD es un grupo heterogéneo de trastornos y de relaciones entre las distintas patologías que conforman este complejo diagnóstico. Debido a la metodología utilizada, a la complejidad diagnóstica que conlleva la distinción entre trastornos mentales primarios y secundarios o inducidos versus independientes y a las dudas sobre la utilidad de estas categorías (Morojele, Saban y Seedat, 2012), estas distinciones no las hemos valorado en este estudio.

\section{Diferencias entre pacientes duales y sujetos con trastornos mentales no duales}

Como hemos señalado existen importantes diferencias sociodemográficas entre ambos grupos, siendo los duales mas parecidos en este sentido a los adictos. Los pacientes duales son más jóvenes, tienen mayor proporción de varones, de convivencia con la familia de origen, de solteros, menor nivel educativo y peor situación laboral que los sujetos con TM no duales. Esto sugiere diferentes factores de riesgo para ambos grupos y mayor gravedad o repercusiones sociolaborales para los pacientes duales, como han constatado otros autores (O'Brien, 2004; Rush y Koegl, 2008).

Otro dato sugerente de mayor gravedad de los trastornos duales que de los TM no duales es el mayor riesgo de suicidio de los primeros, como ya hemos publicado previamente (Szerman et al., 2011) y señalado por distintos autores ya sea referido a trastornos duales en general o para la comorbilidad con ciertos TM específicos (Comtois, Russo, Roy-Byrne y Ries, 2004; Dhossche, Meloukheia, y Chakravorty, 2000; Rush y Koegl, 2008). Además los pacientes duales tenían mayor número de diagnósticos comórbidos del eje I distintos del TUS, casi dos diagnósticos de media, similar a lo referido en otras muestras (Ross et al., 1988).

Respecto a los trastornos mentales del eje I asociados con la presencia de PD hay que destacar principalmente la relación con el trastorno bipolar. Este diagnóstico era 5 veces más frecuente entre los pacientes duales que los no duales. Ya en 1920, Kraepelin reconocía la frecuente comorbilidad entre alcoholismo y psicosis maníaco-depresiva (Brown, 2005). Múltiples trabajos destacan esta asociación como una de las más estrechas considerando un trastorno específico del eje I, con cifras elevadas de prevalencia y elevadas odds ratio de asociación tanto referidas a la manía como cuando se considera en un sentido más amplio el concepto de espectro bipolar (Bauer et al., 2005; Brady y Lydiard, 1992; Brown, 2005; Grant et al., 2004; Kessler, 2004; Levin y Hennessy, 2004). Se ha sugerido que puede existir un sobrediagnóstico debido a que los efectos de ciertas drogas pueden mimetizar los síntomas del trastorno del humor (Brown, 2005). Sin embargo, también es frecuente que se observe que la asociación es mayor con el trastorno bipolar tipo I que con el tipo II o la ciclotimia, los cuáles están sujetos a una mayor confusión diagnóstica (Grant et al., 2004; Levin y Hennessy, 2004). Está descrito que los pacientes con trastorno bipolar primario son un grupo de los que mas prevalencia de TUS presentan (Grant et al., 2004; Levin y Hennessy, 2004; Regier et al., 1990). Por lo tanto, el trastorno bipolar, aún considerando la posibilidad de que hubiera un sobrediagnóstico, es el trastorno del eje I mas ligado a los TUS según nuestros datos, lo que tiene repercusiones diagnósticas (considerar la posibilidad del diagnóstico dual en presencia de uno de ellos, realizar un adecuado diagnóstico diferencial) y terapéuticas (manejo adecuado de ambos trastornos simultáneamente). Además está descrito que la presencia de uno de estos trastornos provoca un peor pronóstico en el otro, con mas recaídas, peores repercusiones funcionales, menor adherencia al tratamiento y mayor riesgo de suicidio (Brown, 2005; Comtois et al., 2004; Levin y Hennessy, 2004).

Dada la naturaleza del estudio no podemos determinar que tipo de relación hay entre estos trastornos. Se ha señalado que el TUS puede precipitar el trastorno bipolar, así está descrita la posibilidad de desencadenar cuadros maníacos o hipomaniacos con drogas como el cannabis o la cocaína. Por otro lado, las alteraciones del estado de ánimo pueden ser factores de riesgo para el consumo, la manía puede favorecer el consumo en relación con la mayor impulsividad, la alteración de la capacidad de juicio o la excesiva implicación en actividades placenteras. Por último, puede haber factores de vulnerabilidad común, factores genéticos, neurobiológicos o trastornos en la infancia que favorezcan ambos (Brown, 2005; Levin y Hennessy, 2004; Winokur et al., 1998).

El trastorno depresivo mayor presentaba una tendencia no estadisticamente significativa a una mayor frecuencia en el grupo no dual. Este trastorno es de los más frecuentes tanto entre pacientes duales como no duales (Blanco et al., 2012; Ross et al., 1988) y aunque algunos trabajos observan la asociación de este trastorno con el diagnóstico dual (Grant et al., 2004), no todos coinciden en ese dato (Lehman et al., 1993). Se ha señalado que la presencia comórbida de ambos puede tener factores de riesgo específicos (Blanco et al., 2012).

El otro grupo de trastornos relacionados con la PD son los trastornos de ansiedad como grupo, hallazgo constatado en la literatura (Grant et al., 2004). De estos, nosotros observamos asociación con los diagnósticos de agorafobia, trastorno por estrés postraumático (TEPT) y trastorno por ansiedad generalizada (TAG), relaciones señaladas por otros autores (Grant et al., 2004; Pietrzak, Goldstein, Southwick y Grant, 2011; Ross et al., 1988). El consumo de drogas se ha considerado que puede servir de automedicación para pacientes fóbicos, fundamentalmente alcohol o cannabis (Robinson, Sareen, Cox y Bolton 2011). El consumo de drogas puede favorecer la exposición a situaciones estresantes y aumentar la vulnerabilidad para el desarrollo de un TEPT tras una situación traumática y el TEPT puede favorecer el consumo de drogas como alivio de ciertos síntomas aunque empeore el curso posterior del trastorno (Fetzner, McMillan, Sareen y Asmundson, 2011; Pietrzak et al., 2011). Los sistemas biológicos implicados en la respuesta al estrés estarían implicados en dicha comorbilidad (Jacobsen, Southwick y Kosten, 2001). El TAG y los TUS son igualmente altamente comórbidos (Alegria et al., 2010). Algunas drogas pueden ocasionar síntomas de ansiedad y de esta forma, los TUS suelen preceder al TAG aunque, después el consumo para el alivio de estos sintomas, puede mantener el cuadro (Alegria et al., 2010). Sin embargo, hay que considerar que es un diagnóstico criticado por su escasa fiabilidad, aún mayor en la población adicta, siendo un diagnóstico cuestionado 0 a veces excluido de los trabajos sobre patología dual (Ross et al., 1988). 
De cualquier forma, la elevada comorbilidad de los síntomas de ansiedad y los TUS tienen repercusiones pronósticas y terapéuticas relevantes, por lo que no pueden ser infravalorados. Esta comorbilidad se asocia con mayor presencia de otros trastornos comórbidos y mayor gravedad y discapacidad asociada (Alegria et al., 2010, Magidson, Liu, Legues y Blanco, 2012).

En contra de lo esperado, no encontramos una asociación del diagnóstico dual con la psicosis. Posiblemente el subgrupo de psicóticos era pequeño para obtener diferencias o dentro de este subgrupo podría haber una sobrerrepresentación de pacientes psicóticos procedentes de CSM que no consumieran drogas. De cualquier forma, hay que destacar que un $11 \%$ de los pacientes duales tenían psicosis, cifra muy superior al porcentaje de pacientes con psicosis que se puede observar en población general o en ámbitos sanitarios distintos de los centros de salud mental y que el $77 \%$ de los pacientes con psicosis tenían un diagnóstico de TUS (59 pacientes con psicosis eran duales y 18 no duales). Esta cifra es similar a la referida sobre prevalencia de TUS entre psicosis por diversos autores (Barnes, Mutsatsa, Hutton, Watt y Joyce, 2006; Westermeyer, 2006).

Los TP estaban sobrerrepresentados en el grupo dual. La prevalencia de estos trastornos era mayor, tenían un mayor número de estos diagnósticos y prácticamente cuando se agrupaban por cluster, cualquiera de estos tres cluster era mas frecuente en la población dual. Esta intensa correlación se ha referido en la amplia literatura al respecto (Compton et al., 2007; Lehman et al., 1993; Weaver et al., 2003).

Considerando TP específicos, la asociación mas intensa fue con el trastorno antisocial de la personalidad (TAP) como ha sido ya descrito (Compton, Conway, Stinson, Colliver y Grant, 2005; Goldstein et al., 2007; Lehman et al., 1993; Limbeck et al., 1992; Ross et al., 1988). Otros trastornos especificos asociados al diagnóstico dual fueron el trastorno límite, a pesar de que este trastorno también es altamente prevalente entre los pacientes con TM no duales como observamos nosotros y señala la literatura (Verheul, 2001), el trastorno paranoide y el esquizoide. En una revisión sobre el tema se señalaron como trastornos más prevalentes en adictos los TAP, límite, evitativo y paranoide (Verheul, 2001). En el estudio NESARC (National Epidemiological Survey on Alcohol and Related Conditions) se observó una asociación de los TUS con el TAP, histriónico, dependiente, paranoide, evitativo o esquizoide (Compton et al., 2007; Grant et al., 2004), aunque ellos no valoraron el trastorno límite, esquizotípico y narcisista. En otro estudio en nuestro medio, los trastornos de personalidad más prevalentes fueron el obsesivo, antisocial, paranoide y dependiente (Echeburúa, De Medina y Aizpiri, 2007).

En general, se considera que ciertos TP favorecen el consumo de drogas, en relación con la mayor impulsividad o el alivio de ciertos sintomas. Otra posibilidad es que el consumo de drogas per se produzca una clínica que mimetiza ciertos TP, por ejemplo conductas antisociales, mayor impulsividad o cambios afectivos que simulen a los trastornos del cluster B. Distintos rasgos de personalidad como el neuroticismo o la búsqueda de novedades se han descrito que pueden contribuir a la comorbilidad (Khan, Jacobson, Gardner, Prescott y Kendler, 2005). Factores genéticos pueden favorecer la comorbilidad entre el TAP y los TUS (Li et al., 2012). En la revisión señalada, se sugie- ren tres mecanismos distintos (desinhibición conductual, reactividad ante el estrés, sensibilidad al refuerzo) por los cuáles se relacionan ciertos TP con determinados TUS y plantean un correlato neurobiológico específico (Verheul, 2001).

El instrumento utilizado para valorar los trastornos de personalidad puede aportar cifras muy dispares de estos, con los autoinformes tendiendo a sobrediagnosticarlos (FernándezMontalvo, Landa, López-Goñi y Lorea, 2006). El cuestionario autoadministrado puede sobredimensionar el diagnóstico de estos trastornos, sin embargo, se producia una comprobación posterior del entrevistador mediante la escala de significación clínica que puede corregir dicha sobrevaloración. De cualquier forma, el mismo instrumento se utilizó para ambos grupos, por lo que la asociación de ciertos TP con el grupo dual no se explica por el instrumento utilizado.

\section{Limitaciones del estudio}

El diagnóstico de TUS se realizó por medio de entrevista clínica, aunque con un instrumento estandarizado. No se incluyó en el protocolo el análisis toxicológico o el uso de otras fuentes de información, sin embargo, la mayoría de los pacientes eran conocidos en los $C D$, donde se realizan habitualmente urinoanálisis y son conocedores de sus patrones de consumo. Es posible la implicación de investigadores más motivados en el estudio de la PD que pudieran sobrediagnosticar el problema, sin embargo, se han usado instrumentos estandarizados, hubo un entrenamiento previo y han participado un alto número de investigadores. No se recogieron datos sobre el consumo activo o la abstinencia de las diversas drogas, el incluir dichas variables hubiera implicado un aumento destacado del tamaño muestral. De cualquier forma, el análisis de estas variables sobre los diagnósticos psiquiátricos se realiza de forma más adecuada con un estudio longitudinal y no transversal como el presente. Como hemos señalado tampoco establecimos distinciones entre diagnósticos primarios o secundarios, inducidos por drogas o no, esto hubiera requerido instrumentos diagnósticos más complejos y mucho mayor tiempo para la valoración, además de las dudas sobre la fiabilidad diagnóstica y las implicaciones pronósticas de estas distinciones. Este estudio muestra algunas asociaciones entre trastornos mentales y adictivos, pero el diseño transversal no permite establecer relaciones causales.

Consideramos que el alto número de investigadores y de centros participantes a lo largo de toda la Comunidad de Madrid con importantes diferencias socioeconómicas es una fortaleza del estudio, dado que los datos pueden ser extrapolables a los sujetos en tratamiento en la red de salud mental y de drogas de esta Comunidad. El uso de una entrevista estructurada y el tamaño muestral obtenido son otras fortalezas del presente estudio. La entrevista estructurada produce mayor validez al diagnóstico de los trastornos mentales en adictos que el diagnóstico clínico habitual (Kranzler et al., 1995).

Este estudio presenta datos relevantes sobre la magnitud del problema de la PD en nuestro medio, las características de estos pacientes, la heterogeneidad de los diagnósticos incluidos en dicho concepto o las relaciones entre ciertos TUS y TM, lo que ayuda para la adecuada detección de estos pacientes y el diag- 
nóstico correcto y orienta para la planificación de los recursos sanitarios que deben de considerar este amplio grupo de población que demanda tratamiento y que presenta unas necesidades diferenciales. Como hemos señalado los datos no son extrapolables a la población general, dado que la comorbilidad favorece la búsqueda de tratamiento, o a otros recursos asistenciales.

\section{Reconocimientos}

Este trabajo fue financiado por la Obra Social Caja Madrid, el Instituto de Adicciones del Ayuntamiento de Madrid, la Agencia Antidroga de la Comunidad de Madrid y la Oficina Regional de Salud Mental de la Comunidad de Madrid. El proyecto fue promovido por la Sociedad Española de Patología Dual.

Los autores expresan sus agradecimientos a todos los investigadores de los CAD, CAID y CSM.

\section{Conflicto de intereses}

El Dr. Szerman es presidente de la Sociedad Española de Patología Dual que fue la promotora del estudio. Los Dres. Arias, Vega, Mesias, Basurte, Ochoa y Poyo eran miembros de la Junta Directiva de esa Sociedad en el momento de realización del estudio. La Dra. Morant trabajaba en la Oficina Regional de Salud Mental que participó en la financiación del estudio. El Dr. Babín era Director del Instituto de Adicciones en el momento del desarrollo del estudio, organismo que participó en la financiación del proyecto.

\section{Referencias}

Agrawal, A., Lynskey, M. T., Madden, P. A., Bucholz, K. K. y Heath, A. C. (2007) A latent class analysis of illicit drug abuse/dependence: results from the National Epidemiological Survey on Alcohol and Related Conditions. Addiction, 102, 94-104.

Alegría, A. A., Hasin, D. S., Nunes, E. V., Liu, S. M., Davies, C., Grant, B. F. y Blanco, C. (2010) Comorbidity of generalized anxiety disorder and substance use disorders: results from the National Epidemiologic Survey on Alcohol and Related Conditions. Journal of Clinical Psychiatry, 71, 1187-95. doi: 10.4088/JCP.09m05328gry.

Barnes, T. R., Mutsatsa, S. H., Hutton, S. B., Watt, H. C. y Joyce, E. M. (2006) Comorbid substance use and age at onset of schizophrenia. British Journal of Psychiatry, 188, 237-42. doi 10.1192/bjp. bp.104.007237.

Bauer, M. S., Altshuler, L., Evans, D. R., Beresford, T., Williford, W. O. y Hauger, R. (2005) Prevalence and distinct correlates of anxiety, substance, and combined comorbidity in a multi-site public sector sample with bipolar disorder. Journal of Affective Disorders, 85, 301-31. doi.org/10.1016/j.jad.2004.11.009.
Blanco, C., Alegría, A. A., Liu, S. M., Secades-Villa, R., Sugaya, L., Davies, C. y Nunes, E. V. (2012) Differences among major depressive disorder with and without co-occurring substance use disorders and substance-induced depressive disorder: results from the National Epidemiologic Survey on Alcohol and Related Conditions. Journal of Clinical Psychiatry, 73, 865-73. doi: 10.4088/JCP.10m06673.

Bowden-Jones, O., Iqbal, M. Z., Tyrer, P., Seivewright, N., Cooper, S., Judd, A. y Weaver, T., COSMIC study team. (2004) Prevalence of personality disorder in alcohol and drug services and associated comorbidity. Addiction, 99, 1306-14.

Brady, K. T. y Lydiard, R. B. (1992) Bipolar affective disorder and substance abuse. Journal of Clinical Psychopharmacology, 12, 17S$22 \mathrm{~S}$.

Brown, E.S. (2005) Bipolar disorder and substance abuse. Psychiatric Clinics of North America, 28, 415-25.

Burns, L. y Teesson, M. (2002) Alcohol use disorders comorbid with anxiety, depression and drug use disorders. Findings from the Australian National Survey of Mental Health and Well Being. Drug and Alcohol Dependence, 68, 299-307. doi.org/10.1016/S03768716(02)00220-X.

Calvo, N., Caseras, X., Gutierrez, F. y Torrubia, R. (2002). Spanish version of the personality diagnostic questionnaire-4+ (PDQ-4+). Actas Españolas de Psiquiatría, 30, 7-13.

Chan, Y. F., Dennis, M. L. y Funk, R. R. (2008) Prevalence and comorbidity of major internalizing and externalizing problems among adolescents and adults presenting to substance abuse treatment. Journal of Substance Abuse and Treatment, 34, 14-24. doi. org/10.1016/j.jsat.2006.12.031.

Compton, W. M., Conway, K. P., Stinson, F. S., Colliver, J. D. y Grant, B. F. (2005) Prevalence, correlates, and comorbidity of DSM-IV antisocial personality syndromes and alcohol and specific drug use disorders in the United States: results from the national epidemiologic survey on alcohol and related conditions. Journal of Clinical Psychiatry, 66, 677-85.

Compton, W. M., Thomas, Y. F., Stinson, F. S. y Grant, B. F. (2007). Prevalence, correlates, disability, and comorbidity of DSMIV drug abuse and dependence in the United States: results from the national epidemiologic survey on alcohol and related conditions. Archives of General Psychiatry, 64, 566-76. doi:10.1001/ archpsyc.64.5.566.

Comtois, K. A., Russo, J. E., Roy-Byrne, P. y Ries, R. K. (2004) Substance Abuse as Predictors of Suicidal Behavior in Acutely Hospitalized Psychiatric Inpatients. Biological Psychiatry, 56, 757-763.

Dhossche, D. M., Meloukheia, A. M. y Chakravorty, S. (2000) The Association of Suicide Attempts and Comorbid Depression and Substance Abuse in Psychiatric Consultation Patients. General Hospital Psychiatry, 22, 281-288. doi.org/10.1016/S01638343(00)00085-2.

Dragt, S., Nieman, D. H., Schultze-Lutter, F., van der Meer, F., Becker, H., de Haan, L.... Linszen, D. H. (2012) Cannabis use and age at onset of symptoms in subjects at clinical high risk for psychosis. Acta Psychiatrica Scandinavica, 125, 45-53. doi: 10.1111/j.16000447.2011.01763.x. 
Echeburúa, E., De Medina, R. B. y Aizpiri, J. (2007) Comorbidity of alcohol dependence and personality disorders: a comparative study. Alcohol and Alcoholism, 42, 618-22. doi: 10.1093/alcalc/agm050.

Fernández-Montalvo, J., Landa, N., López-Goñi, J. J. y Lorea, I. (2006) Personality disorders in alcoholics: a comparative pilot study between the IPDE and the MCMI-II. Addictive Behavior, 31, 1442-8. doi.org/10.1016/j.addbeh.2005.09.014.

Fetzner, M. G., McMillan, K. A., Sareen, J. y Asmundson, G. J. (2011) What is the association between traumatic life events and alcohol abuse/ dependence in people with and without PTSD? Findings from a nationally representative sample. Depression and Anxiety, 28, 632-8. doi: 10.1002/da.20852.

Goldstein, R. B., Compton, W. M., Pulay, A. J., Ruan, W. J., Pickering, R. P., Stinson, F. S. y Grant, B. F. (2007) Antisocial behavioral syndromes and DSM-IV drug use disorders in the United States: results from the National Epidemiologic Survey on Alcohol and Related Conditions. Drug and Alcohol Dependence, 90, 145-58. doi: 10.1016/j.drugalcdep.2007.02.023

González-Saiz, F., Lozano, O. M., Martín, J., Bilbao, I., Ballesta, R., Gutiérrez, J. (2011) Comorbilidad psiquiátrica en una muestra de pacientes con dependencia de opiáceos tratados con buprenorfina sublingual en régimen de comunidad terapéutica. Revista de Psiquiatría y Salud Mental, 04, 81-7.

Grant, B. F., Stinson, F. S., Dawson, D. A., Chou, S. P., Dufour, M. C., Compton, W.... Kaplan, K. (2004) Prevalence and co-occurrence of substance use disorders and independent mood and anxiety disorders: results from the National Epidemiologic Survey on Alcohol and Related Conditions. Archives of General Psychiatry, 61, 807-16. doi:10.1001/archpsyc.61.8.807.

Gual, A. (2007). Dual Diagnosis in Spain. Drug and Alcohol Review, 26, 65-71.

Jacobsen, L. K., Southwick, S. M. y Kosten, T. R. (2001) Substance use disorders in patients with posttraumatic stress disorder: a review of the literature. American Journal of Psychiatry, 158, 1184-90. doi 10.1176/appi.ajp.158.8.1184

Kandel, D. B., Huang, F. Y. y Davies, M. (2001) Comorbidity between patterns of substance use dependence and psychiatric syndromes. Drug and Alcohol Dependence, 64, 233-241. doi.org/10.1016/S03768716(01)00126-0.

Kessler, R. C. (2004) The epidemiology of dual diagnosis. Biological Psychiatry, 56, 730-7. .doi.org/10.1016/j.biopsych.2004.06.034.

Kessler, R. C., Chiu, W. T., Demler, O., Merikangas, K. R. y Walters, E. E. (2005). Prevalence, severity, and comorbidity of 12-month DSM-IV disorders in the National Comorbidity Survey Replication. Archives of General Psychiatry, 62, 617-27. doi:10.1001/archpsyc.62.6.617.

Khan, A. A., Jacobson, K. C., Gardner, C. O., Prescott C. A. y Kendler K. S. (2005) Personality and comorbidity of common psychiatric disorders. British Journal of Psychiatry, 186, 190-196. doi 10.1192/ bjp.186.3.190.

Kranzler, H. R., Kadden, R. M., Burleson, J. A., Babor, T. F., Apter, A. y Rounsaville B. J. (1995) Validity of psychiatric diagnoses in patients with substance use disorders: is the interview more important than the interviewer? Comprehensive Psychiatry, 36, 278-88. doi. org/10.1016/S0010-440X(95)90073-X.
Lehman, A. F., Myers, C. P., Thompson, J. W. y Corty, E. (1993) Implications of mental and substance use disorders. A comparison of single and dual diagnosis patients. Journal of Nervous and Mental Diseases, 181, 365-70.

Levin, F. R. y Hennessy, G. (2004) Bipolar disorder and substance abuse. Biological Psychiatry, 56, 738-48. doi.org/10.1016/j. biopsych.2004.05.008.

Li, D, Zhao, H., Kranzler, H. R., Oslin, D., Anton, R. F., Farrer, L. A. y Gelernter, J. (2012) Association of COL25A1 with Comorbid Antisocial Personality Disorder and Substance Dependence. Biological Psychiatry, 71, 733-740.

Magidson, J. F., Liu, S. M., Legues, C. W. y Blanco, C. (2012) Comparison of the course of substance use disorders among individuals with and without generalized anxiety disorder in a nationally representative sample. Journal of Psychiatric Research, 46, 659-66. doi: 10.1016/j. jpsychires.2012.02.011.

Marsden, J., Gossop, M., Stewart, D., Rolfe, A. y Farrell, M. (2000) Psychiatric symptoms among clients seeking treatment for drug dependence. Intake data from the National Treatment Outcome Research Study. British Journal of Psychiatry, 176, 285-9. doi: 10.1192/bjp.176.3.285.

Morojele, N. K., Saban, A. y Seedat, S. (2012) Clinical presentations and diagnostic issues in dual diagnosis disorders.. Current Opinion in Psychiatry, 25, 181-6. doi: 10.1097/YC0.0b013e328351a429.

O'Brien, C. P., Charney, D. S., Lewis, L., Cornish, J. W., Post, R. M., Woody, G. E. ... Weisner, C. (2004) Priority actions to improve the care of persons with co-ocurring substance abuse and other mental disorders: A call to action. Biological Psychiatry, 56, 703-713. doi:10.1016/j.biopsych.2004.10.002.

Pedrero-Pérez, E. J., Puerta-García, C., Lagares, A. y Sáez-Maldonado, A. (2003) Prevalencia e intensidad de los trastornos de personalidad en adictos a sustancias en tratamiento en un centro de atención a las drogodependencias. Trastornos Adictivos, 5, 241-255.

Pietrzak, R. H., Goldstein, R. B., Southwick, S. M. y Grant, B. F. (2011) Prevalence and Axis I comorbidity of full and partial posttraumatic stress disorder in the United States: results from Wave 2 of the National Epidemiologic Survey on Alcohol and Related Conditions. Journal of Anxiety Disorders, 25, 456-65. doi: 10.1016/j. janxdis.2010.11.010.

Regier, D. A., Farmer, M. E., Rae, D. S., Locke, B. Z., Keith, S. J., Judd, L. L. y Goodwin, F. K. (1990). Comorbidity of mental disorders with alcohol and other drug abuse. Results from the Epidemiologic Catchment Area (ECA) Study. JAMA, 264, 2511-8. doi:10.1001/ jama.1990.03450190043026.

Robinson, J., Sareen, J., Cox, B. J. y Bolton, J. M. (2011) Role of selfmedication in the development of comorbid anxiety and substance use disorders: a longitudinal investigation. Archives of General Psychiatry, 68, 800-7. doi: 10.1001/archgenpsychiatry.2011.75.

Ross, H. E., Glaser, F. B. y Germanson, T. (1988) The prevalence of psychiatric disorders in patients with alcohol and other drug problems. Archives of General Psychiatry, 45, 1023-31. doi:10.1001/ archpsyc.1988.01800350057008.

Rush, B. y Koegl, C. J. (2008) Prevalence and profile of people with co-occurring mental and substance use disorders within a 
comprehensive mental health system. Canadian Journal of Psychiatry, 53, 810-21.

Sheehan, D. V., Lecrubier, Y., Sheehan, K. H., Amorim, P., Janavs, J., Weiller, E. ... Dunbar, G. C. (1998) The Mini-International Neuropsychiatric Interview (M.I.N.I.): the development and validation of a structured diagnostic psychiatric interview for DSM-IV and ICD-10. Journal of Clinical Psychiatry, 59 Suppl 20, 22-33.

Szerman, N., Arias, F., Vega, P., Babin, F., Mesias, B., Basurte, I. ... Poyo, F. (2011) Estudio piloto sobre la prevalencia de patología dual en pacientes en tratamiento en la Comunidad de Madrid. Adicciones, 23, 249-256.

Szerman, N., Lopez-Castroman, J., Arias, F., Morant, C., Babin, F., Mesias, B., ... Baca-García, E. (2011) Dual Diagnosis and Suicide Risk in a Spanish Outpatient Sample. Substance Use \& Misuse, 48, 1-7. doi: 10.3109/10826084.2011.636135.

Torrens, M., Gilchrist, G. y Domingo-Salvany, A. (2011) Psychiatric comorbidity in illicit drug users: Substance-induced versus independent disorders. Drug and Alcohol doi: 10.1016/j. drugalcdep.2010.07.013. Dependence, 113, 147-156. doi: 10.1016/j. drugalcdep.2010.07.013.

Vergara-Moragues, E., González-Saiz, F., Lozano, O. M., Betanzos, P., Fernández, F., Bilbao-Acebos, I., Pérez, M., Verdejo, A. (2012) Psychiatric comorbidity in cocaine users treated in therapeutic community: Substance-induced versus independent disorders. Psychiatry Research, 200, 734-41. doi: 10.1016/j. psychres.2012.07.043.

Verheul, R. (2001) Co-morbidity of personality disorders in individuals with substance use disorders. European Psychiatry, 16, 274-82. doi. org/10.1016/S0924-9338(01)00578-8.

Volkow, N. D (2004). The reality of comorbidity: depression and drug abuse. Biological Psychiatry, 56, 714-7. doi:10.1016/j. biopsych.2004.07.007.

Volkow, N. D (2007). Addiction and Co-ocurring mental disorders. Director's perspective. NIDA Notes, 21, 2.

Weaver, T., Madden, P., Charles, V., Stimson, G., Renton, A., Tyrer, P. ... Ford, C. (2003). Comorbidity of substance misuse and mental illness in community mental health and substance misuse services. British Journal of Psychiatry, 183, 304-313. doi: 10.1192/02-623.

Westermeyer, J. (2006) Comorbid schizophrenia and substance abuse: a review of epidemiology and course. American Journal of Addictions, $15,345-55$.

Winokur, G., Turvey, C., Akiskal, H., Coryell, W., Solomon, D., Leon, A. ... Keller, M. (1998) Alcoholism and drug abuse in three groups-bipolar I, unipolars and their acquaintances. Journal of Affective Disorders, 50, 81-9. 